\title{
Digital health technologies for universal health coverage: a promising change
}

\author{
Prakash Babu Kodali* and Shankar Das
}

Digital health technologies act as health system strengthening tools and facilitate achievement of universal health coverage (UHC). In line with the United Nations sustainable Development Goals, India launched the Ayushman Bharat Programme (ABP) to strengthen health services and achieve UHC. Digital health technologies could be instrumental in improving the efficiency of ABP. Given that India is in the initial steps towards UHC and digital health deployment, synergy between health programmes and digital health policies is essential to realize the goal of UHC. This article aims to discuss the scope of digital health technologies for achieving UHC, their application, opportunities and challenges in the context of ABP in India.

Keywords: Digital health technologies, programmes and policies, sustainable development goals, universal health coverage.

THE ongoing COVID-19 pandemic overstretched the health systems in developed and developing countries alike. The losses attributable to the pandemic undoubtedly impacted on the achievement of sustainable development goals (SDGs) set for 2025. The pandemic also provided a window of opportunities to leverage existing information technology (IT) solutions and find new ones. These IT solutions can facilitate in the achievement of SDGs, specifically those concerning health. One of relevant targets of SDGs in this regard is achievement of universal health coverage (UHC). The crucial aim of UHC is to safeguard access to basic healthcare services for everyone and everywhere without facing financial hardships. Growing evidence indicates that UHC achievement can be greatly leveraged by digital health technologies ${ }^{1}$.

'Digital health' is a broader term which encompasses utilization of information, communication and electronic technologies for improvement of health and providing healthcare. The technologies used in healthcare could vary from simple hand-held mobile phones/wearables to highly advanced satellite technologies, enabling the health systems to collect, record and transmit health information, facilitate decision-making and provide healthcare. Digital health technologies have extended an abundance of possibilities for shaping the future of UHC, and ensuring effective public health policy and programmes.

With the much-lauded launch of Ayushman Bharat Programme (ABP) and implementation of current policies

Prakash Babu Kodali is in the Department of Public Health and Community Medicine, Central University of Kerala, Kasaragod 671316 , India and Shankar Das is in the International Institute of Health Management Research, New Delhi 110 075, India.

*For correspondence. (e-mail: prakashkodali@cukerala.ac.in) in the country, India's commitment towards achieving UHC is clearly reflected. The Indian health system is currently in a phase of transition towards UHC. Interestingly, the Indian healthcare system is also in a transformative phase with the deployment of digital health technology. The literature reports growing prominence of digital health technologies in improving healthcare delivery at the individual, primary, secondary and tertiary healthcare levels. Table 1 lists some of the digital health technologies commonly used. Recently, the Government of India (GoI) launched the National Digital Health Mission (NDHM) to facilitate the usage of digital health technologies in strengthening healthcare delivery. Given that digital health interventions hold the potential to improve efficiency of healthcare provision, we argue that synergy between both the components, i.e. health programmes and digital health policies of the country is much needed to realize UHC. This article aims to build a perspective for digital health in the Indian scenario and discuss the wider scope for digital health technologies in realizing the goal of UHC in India. Though the need for a robust IT system in healthcare has been well recognized, there is an unclear picture on implications of digital health technologies in the larger context of UHC in India, the opportunities for digital health and the possible challenges. This article further discusses the interlocking perspectives of digital health and UHC within the context of ABP.

\section{Ayushman Bharat and the road to UHC in India}

The initiative of the Pradhan Mantri - the Jan Arogya Yojana or ABP is considered as India's giant leap towards achieving UHC. ABP envisions to provide comprehensive 
Table 1. Common digital health technologies available at various levels of healthcare

\begin{tabular}{ll}
\hline Level of care & \multicolumn{1}{c}{ Digital health technology } \\
\hline Individual level & Wearables \\
& Point-of-care devices \\
& Teleconsultations \\
& Interactive voice response system-based approaches \\
& Health chatbots \\
& Mobile health applications \\
& Mobile health technologies \\
& Portable medical diagnostics \\
& Telehealth services \\
& Community/family health records \\
& Mobile device management applications \\
& Clinical decision support systems \\
& Medicine and vaccine supply chain management applications \\
& Artificial intelligence-based disease monitoring \\
& Electronic health records \\
& Hospital management systems \\
Telehealth services & Clinical decision support systems \\
Digital diagnostics & Digital pharmacy \\
& Robotics \\
\hline &
\end{tabular}

healthcare services (primary, secondary and tertiary) through Health and Wellness Centres (HWCs), public health facilities and empanelled hospitals. The programme under its National Health Protection Scheme (NHPS) component aims to cover around 500 million population (i.e. 100 million families). Each family is covered a sum of INR 500,000 per year and can avail health services in the empanelled hospitals across the country with completely cashless treatment for over 1300 different procedures ${ }^{2}$. Additionally, ABP envisions to strengthen the existing health system and provide comprehensive primary healthcare through upgradation of sub-centres and primary health centres (PHCs) into $\mathrm{HWCs}^{3}$. The HWCs emphasize on health promotion and prevention of ill-health by keeping communities healthy, by engaging and empowering them to choose healthy lifestyle and adopt behaviours that reduce the risk of developing chronic diseases and morbidities.

\section{The potential of digital health technologies in health service provision}

The last two decades have seen a sharp rise in the use of digital health technologies in health service provision. Telemedicine, electronic medical records, mobile health applications, point-of-contact devices, GPS systems and clinical decision-making systems have improved the timeliness and quality of healthcare services ${ }^{4}$. Moreover, evidence also suggests that the seemingly non-health technologies like mobile instant messaging applications (such as WhatsApp) have been innovatively used to improve healthcare delivery ${ }^{5}$. While countries are at various stages of digital health technology diffusion, the Indian experience with digital health is largely led by funded projects and research studies, or confined to selected tertiary healthcare centres and big private hospitals. However, evidence suggests that digital health technologies have been largely effective in the Indian context.

The use of digital health technologies reduced maternal and neonatal deaths ${ }^{6}$, improved quality of care $^{7}$, facilitated clinical decision-making ${ }^{8}$, improved management of Non Communicable Diseases (NCDs) ${ }^{8-10}$, enabled health worker training ${ }^{11}$, improved data management ${ }^{12}$ and facilitated provision of psychological services ${ }^{13}$.

Integration of digital health technologies to the formal health system is relatively new in India and is being actively taken up. Development of standards for electronic medical records (EMR), fostering the use of mHealth technologies (such as ANMOL, eAushadi, etc.), and the recent launch of NDHM reflect the growing prominence of digital health technologies in the Indian healthcare ecosystems.

\section{Digital health: instrument for strengthening UHC}

Many states have constructively engaged and strongly pitched to expand the coverage of ABP (it now covers 130 million families) with strong leadership and readiness to adopt UHC as their primary health goal ${ }^{14}$. The scale at which ABP is currently implemented, invites innovative means to enable its successful implementation. The provisioning of healthcare services under ABP could be characterized into two aspects: (i) Primary healthcare through HWCs and (ii) secondary and tertiary healthcare 


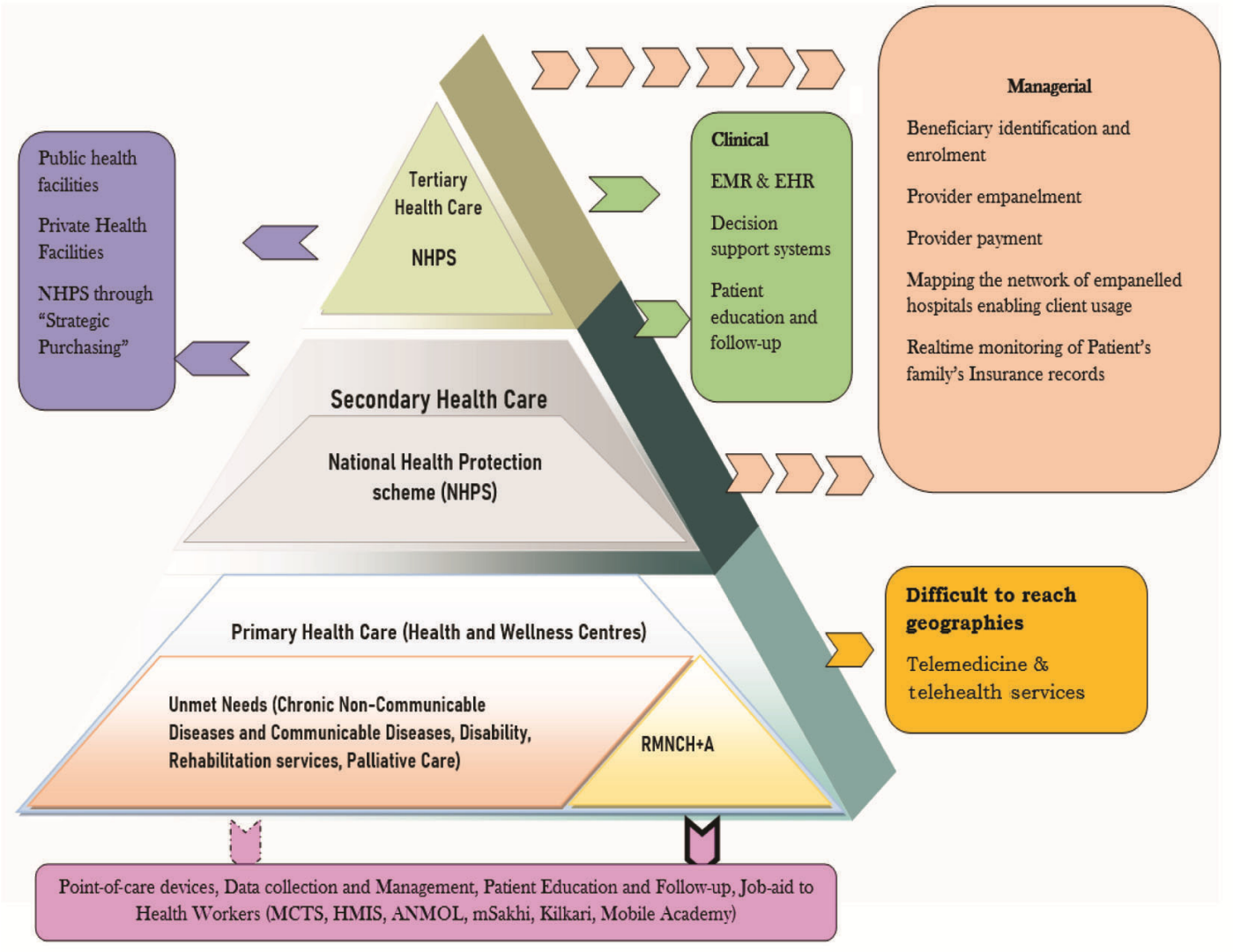

Figure 1. Framework for deployment of digital health technologies in the context of Ayushman Bharat Programme.

through health facilities empanelled in NHPS ${ }^{15}$. Based on the figure developed by Lahariya (2018), a framework was designed to discuss the application of digital health technologies in the context of Ayushman Bharat Programme. The framework was given in Figure 1 and specific details of the same are discussed below.

\section{Primary healthcare through HWCS}

GoI envisaged provisioning comprehensive healthcare, including (a) reproductive, maternal, new-born, child and adolescent health $(\mathrm{RMNCH}+\mathrm{A})$, (b) communicable diseases and (c) non-communicable diseases through 150,000 HWCs across the country. Out of over 0.2 million existing frontline health centres (primary health centres (PHCs) and sub-centres), 0.15 million will be upgraded into HWCs in addition to the already existing public health facilities ${ }^{15}$. The HWCs are expected to act as the first-line centres for providing essential healthcare. Considering their apparent role in primary healthcare provision, HWCs could act as sites for implementation of various digital health initiatives (both existing and new). Similarly, digital technologies can aid in efficient functioning of $\mathrm{HWCs}^{16}$. The application of digital health technologies at the level of health and wellness centres is manyfold.
HWCs as telemedicine centres: The upgradation of existing PHCs into HWCs will provide an opportunity for essential and specialist services in the under-served areas. Specifically, identified HWCs could serve as telemedicine centres in difficult-to-reach geographies, remote rural areas and tribal districts which do not have tertiary healthcare facilities. Moreover, developing selected HWCs as telemedicine centres could be an immediate solution for the shortage of specialists in the rural healthcare ecosystem ${ }^{17}$. The experiences of eUrban PHCs in Andhra Pradesh reflect the potential for HWCs to be the centres for telemedicine ${ }^{18}$.

mHealth technologies for data management and service provision: Existing evidence reports mHealth technologies to be effective in health data management and service provision. Currently, several mHealth technologies exist in the domain of reproductive and child health (ANMOL, mSakhi, Matha Sisu Samrakshana to name few). Despite existing evidence there is limited scale-up of mHealth technologies for NCD screening, identification of high-risk individuals, health education and followup. Integrating the multitude of existing mHealth applications and developing holistic applications, in line with the larger objectives of HWCs, could effectively improve the health service delivery. Moreover, mHealth applications can also aid in encouraging physical activity, monitoring 


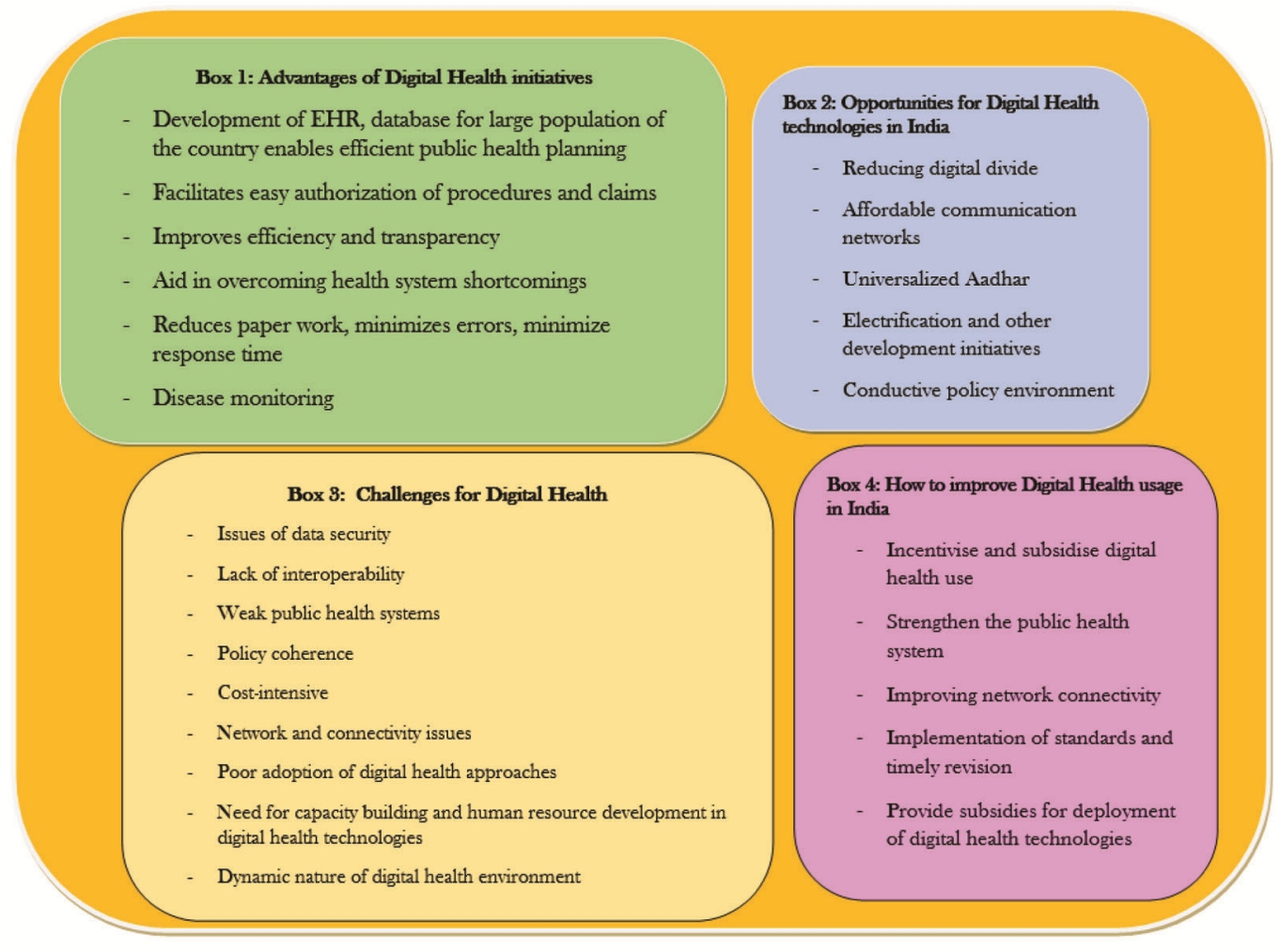

Figure 2. Outline of advantages, opportunities and challenges for digital health technologies.

dietary intake, and tracking the medication adherence among individuals with chronic illness.

Point-of-care devices - taking health care to the community: In addition to the information and communication technologies, the point-of-care devices will improve healthcare access by taking healthcare to the community. Timely access to essential healthcare services such as laboratory testing, monitoring and imaging could be provided within the community setting ${ }^{19}$. The affordability and accuracy of point-of-care devices (such as glucometers, automatic blood pressure monitoring devices, portable pulse oximeters, etc.) make them extremely relevant for community-based screening and management of NCDs. Moreover, such devices could also facilitate provision of palliative care at the community level, particularly considering the growing burden of NCDs such as cancer and stroke.

Additionally, interactive voice response system (IVRS)-based digital health applications could enable delivery of essential health education to patients and their follow-up during the course of service provision. Also, HWCs act as primary contact points and referral centres.

Several cadres of frontline health workers, such as multipurpose health workers, Accredited Social Health Activists (ASHA) and mid-level care providers work at the level of $\mathrm{HWCs}^{15}$. Empowering them with digital health devices and providing sufficient training will ena- ble them to function optimally. Moreover, digital health technologies (involving mobile instant messaging or mobile device management applications) can also facilitate the monitoring and supervision of frontline health workers in community settings.

\section{NHPS: towards secondary and tertiary healthcare}

The NHPS launched under ABP aims to provide cashless access to secondary and tertiary healthcare services to all beneficiaries of the scheme. Digital health technologies could improve efficiency of providing the services through $\mathrm{NHPS}^{20}$. Some of the important technologies that could be used in the context of NHPS are discussed below.

Electronic medical records: The electronic medical records (EMRs) help in systematic collection and storing of electronic health information of patients. They will enable the healthcare providers to electronically maintain the healthcare data (diagnosis, diagnostic tests and hospitalization). Considering the risk of supplier-induced demand and moral hazards, EMRs could minimize such agency problems in NHPS by improving transparency. Additionally, they may enable real-time monitoring of the beneficiary and settlement of health insurance claims. The GoI's digitization initiatives such as integrated health 
information programme, the EMR standards, and Aadhaar (unique identification number) serve as opportunities for initiation of the use of EMR technologies.

Hospital Management Systems: The hospital management systems (HMS) are software which facilitate in the functioning of hospitals, encompassing multiple functions such as patient management, human resource management, billing, supplies management, etc. ${ }^{21}$. Encouraging use of HMS will specifically enable the government to identify private health facilities with a specified set of resources, thereby enabling the strategic purchasing envisaged in recent policy documents. HMS have the potential to ensure evidence-based purchasing enabling efficient utilization of public health resources.

GIS mapping technologies: Knowledge about empanelled health facilities in a region is one of the key determinants of optimum utilization of health insurance schemes. Mapping the empanelled health facilities using GIS mapping will enable the beneficiaries to identify the nearest empanelled hospitals, thereby reducing travel time and cost.

mHealth technologies: These have the potential for wider application in the context of NPHS. An mHealth application integrating multiple deliverables could act as a health system strengthening tool. The mHealth technologies could help beneficiaries to track the status of their coverage, identify the empanelled hospital, identify the services provided and track their claim status. In addition, mHealth technologies enable immediate and reliable patient feedback mechanisms so as to improve health services through NHPS.

The digital health technologies can aid in multiple phases of NHPS implementation such as beneficiary identification, empanelment of hospitals, resource allocation and strategic purchasing, and data management. More specifically, digital health technologies can enable efficient monitoring of healthcare provision, as reported by a multitude of eHealth literature ${ }^{22}$. Figure 2 outlines the advantages and opportunities of digital health technologies.

\section{NDHM: a much-needed initiative}

The recent launch of NDHM reverberates the policy-level commitment to strengthening Indian health system by leveraging digital health technologies ${ }^{23}$. NDHM focuses on digitalizing health records through Health ID, Digi Doctor, Health Facility Registry (HFR), Personal Health Records (PHR) and EMRs ${ }^{23}$. In a broader sense, NDHM will enable digitization of individual medical history, data integration and standardization. It could potentially enable doctors to access complete medical history of an individual in just one computer click. Moreover, initiatives under NDHM can actively facilitate tackling of growing burden of NCDs by strengthening service provision in public health facilities.

As the country's health system confronts the growing burden of NCDs, persistent emerging and remerging infectious diseases, widespread health inequities, poor healthcare access, infrastructural inadequacies, shortage of trained manpower and high out of pocket health care expenses, the launch of NDHM is a timely initiative to leverage the potential of digital health technologies to address the persisting challenges. Moreover, being launched two years after the ABP, NDHM can potentially facilitate the development of necessary digital health infrastructure to strengthen ABP.

However, though in the initial stages, the shortcomings of NDHM are to be noted and addressed. NDHM is critiqued for its unilateral focus on developing a digital health records and ignoring the need for strengthening the health system without which digital health records are of little value $^{24}$. While it could be said that digital health records planned to be developed by NDHM enable NHPS under ABP to be more efficient, it is unclear how NDHM will strengthen HWCs which are an equally important component of ABP. Over 51\% of the total healthcare expenditure in India is spent towards primary healthcare. However, the focus of NDHM is more inclined towards secondary and tertiary healthcare. Majority of the healthcare at the grassroots is provided by individual physicians, clinics and nursing homes. Enabling them to invest in health IT and adopt to EMR systems is challenging. Additionally, there could be disruption of normal clinical care, dual data entry (both in electronic and paper-based systems), and burdening of health workers which could hinder the adoption of IT systems.

Prior experiences from developed countries where similar digital health policies were implemented reported that lack of adoption among providers, infrastructural challenges, inadequate training, lack of interoperability and disruption of clinical practice were predominant challenges ${ }^{25}$. The Indian healthcare ecosystem is complex with a large number of healthcare providers (both public and private) at various levels across the systems of medicine, underfunded public health systems, state and regional variations in health, economic and digital divide across states and serving over a billion population. Given the complexities, one should anticipate that the above challenges might amplify and deter the progress to be made.

Nevertheless, the launch of NDHM is a much-needed initiative to facilitate ABP in paving the path for UHC in India. Increasing the public spending on health to at least $2.5 \%$ of GDP, addressing health worker shortage, investments in health infrastructure, capacity building among health workers and bringing awareness among patients and providers are essential for the success of ABP and NDHM. 


\section{Implementation of digital health technology: challenges}

Though the application of digital health technologies enables efficient health service delivery and hastens the progress towards achieving UHC, multiple challenges exist for successful implementation of digital health initiatives (Figure 2).

Currently, the diffusion and adoption of digital health technologies are comparatively less in the Indian context ${ }^{1}$. With respect to technologies such as EMRs, challenges persist in relation to infrastructure, maintenance and human resource. Installation and management of HMS and EMR systems require financial and human resource investments, and are often cost-intensive ${ }^{26}$. Only a limited number of private and public hospitals have begun implementing HMS and EMR systems in India. While the pace at which these systems are implemented is slow, it could be improved by conductive policies and incentives. A specific example could be cited from the 'Affordable Care Act' in the United States, where EMR adoption was given priority for improving the quality of care and cutting down the $\operatorname{costs}^{27}$. Similar prioritization could be followed in the Indian scenario, by providing subsidies and incentives to the hospitals for initiating HMS and EMR systems.

The mHealth technologies are the most commonly used digital health technologies in India. Multiple mHealth technologies are currently running as research projects, pilots, and government-led projects in the areas of maternal and child health, NCD prevention and management, and health service delivery. Majority of mHealth interventions are mobile-based applications, and face the challenge of acceptability and sustained usage ${ }^{28}$. Moreover, presence of too many mHealth IT systems with limited interoperability poses significant challenges.

Lack of IT-related manpower and limited capacity of the health workers to use digital health technologies could be a significant barrier to digital health implementation. Also, partial integration of digital health technologies can result in parallel use of paper-based and digital systems, overburdening the health workers. Lack of interoperability between the digital technologies can cause inconsistencies and data loss. Poor connectivity is another prominent challenge observed for successful implementation of digital health strategies, including the recent ones like eSanjeevaniOPD ${ }^{29}$. Additionally, the issues of privacy, data security and lack of trust regarding digital health systems compromise digital health adoption.

\section{Conclusion}

Recognizing the importance of conductive environment in the light of digital health technologies that may enable in attaining UHC particularly in the context of India's
ABP, the scope of digital health technologies is diverse with applications in almost all the phases of initiation, implementation and evaluation. For a comprehensive UHC like ABP, data management systems, automation and interoperability are the need of the day, which could be addressed by digital health technologies and the recent NDHM. While the opportunities are ample, the deployment of these technologies is faced with slow diffusion, limited adoption, digital illiteracy, technical, human resource and financial challenges. A sustained conductive policy environment with financial investments and incentives will advance the diffusion of digital technologies in improving overall efficiency of the health system in India.

1. WHO, Global diffusion of eHealth: making universal health coverage achievable: report of the third global survey on eHealth, World Health Organization, Geneva, 2016.

2. NHA, Ayushman Bharat Pradhan Mantri Jan Arogya Yojana: Beneficiary Empowerment Guidebook, National Health Authority, 2018.

3. Bakshi, H., Sharma, R. and Kumar, P., Ayushman Bharat Initiative (2018): what we stand to gain or lose! Indian J. Community Med.: Off. Publ. Indian Assoc. Prevent. Soc. Med., 2018, 43, 6366; doi:10.4103/ijcm.IJCM_96_18.

4. Labrique, A. et al., WHO Digital Health Guidelines: a milestone for global health. npj Digit. Med., 2020, 3, 1-3.

5. Pahwa, P., Lunsford, S. and Livesley, N., Experiences of Indian health workers using WhatsApp for improving aseptic practices with newborns: exploratory qualitative study. JMIR Med. Informat., 2018, 6, e13.

6. Prinja, S. et al., Cost effectiveness of mHealth intervention by community health workers for reducing maternal and newborn mortality in rural Uttar Pradesh, India. Cost Effect. Resour. Allocation, 2018, 16, 25.

7. Kaphle, S. et al., Adoption and usage of mHealth technology on quality and experience of care provided by frontline workers: observations from rural India. JMIR mHealth and uHealth, 2015, 3, e61.

8. Praveen, D. et al., SMARTHealth India: development and field evaluation of a mobile clinical decision support system for cardiovascular diseases in rural India. JMIR mHealth and uHealth, 2014,2 , e54.

9. Pfammatter, A. et al., mHealth intervention to improve diabetes risk behaviors in India: a prospective, parallel group cohort study. J. Med. Internet Res., 2016, 18, e207.

10. Hamine, S. et al., Impact of mHealth chronic disease management on treatment adherence and patient outcomes: a systematic review. J. Med. Internet Res., 2015, 17, e52.

11. Modi, D. et al., mHealth intervention 'ImTeCHO' to improve delivery of maternal, neonatal, and child care services - a clusterrandomized trial in tribal areas of Gujarat, India. PLoS Med., 2019, 16, e1002939.

12. Venkat, P. G., Intermediate assessment of the Khushi Baby Crct: Implementation of a novel mHealth solution for vaccination record keeping in rural Udaipur, Rajasthan, India, 2016.

13. Khan, A. et al., Design and development of a digital program for training non-specialist health workers to deliver an evidence-based psychological treatment for depression in primary care in India. J. Technol. Behav. Sci., 2020, 5(4), 1-14.

14. Aman, S., Ayushman CEO seeks universal health cover. Economic Times, 2019; https://economictimes.indiatimes.com/news/politicsand-nation/ayushman-ceo-seeks-universal-health-cover/articleshow/ 72971565.cms (accessed on 6 November 2020). 
15. Lahariya, C., 'Ayushman Bharat' Program and universal health coverage in India. Indian Pediatr., 2018, 55, 495-506.

16. Nair, P., The emerging concept of an inclusive mHealth ecosystem in India. In Emerging Trends and Innovations in Privacy and Health Information Management, IGI Global, 2019, pp. 116-141.

17. Singh, A., Shortage and inequalities in the distribution of specialists across community health centres in Uttar Pradesh, 2002-2012. BMC Health Serv. Res., 2019, 19, 331.

18. GoVAP. e Urban Primary Health Centres, 2020; http://euphcap-gov.in/ (accessed on 24 September 2020).

19. Price, C. P. and Kricka, L. J., Improving healthcare accessibility through point-of-care technologies. Clin. Chem., 2007, 53, 16651675.

20. Gopichandran, V. et al., Ethical challenges of digital health technologies: Aadhaar, India. Bull. WHO, 2020, 98, 277.

21. White, G. et al., Patient medication IV delivery pump with wireless communication to a hospital information management system. United States patent US 6,790,198, 14 September 2004.

22. Kant, L. and Krishnan, S. K., Information and communication technology in disease surveillance, India: a case study. BMC Public Health, 2010, 10, S1-S11; doi:10.1186/1471-2458-10-S1-S11.

23. NDHM, National Digital Health Mission, 2020; https:// ndhm.gov.in/ (accessed on 1 October 2020).
24. Ananthakrishnan, Can a digital ID aid India's primary health ecosystem? 2020; https://www.thehindu.com/opinion/op-ed/can-adigital-id-aid-indias-primary-health-ecosystem/article32460458. ece (accessed on 6 November 2020).

25. Bajpai, N. and Wadhwa, M., India's National Digital Health Mission. Center for Sustainable Development, Columbia University, USA, 2020.

26. Srivastava, S. K., Adoption of electronic health records: a roadmap for India. Healthcare Informat. Res., 2016; 22, 261-269; doi:10.4258/hir.2016.22.4.261.

27. Fontenot, S. F., The affordable care act and electronic health care records: can technology help reduce the cost of health care? Physician Executive, 2014, 40, 68.

28. Pohl, M., mHealth App Economics 2017/2018: Current Status and Future Trends in Mobile Health, 2017.

29. https://play.google.com/store/apps/details?id=in.hied.esanjeevaniopd\&hl=en_US\&showAllReviews $=$ true $($ accessed on 30 September 2020).

Received 23 July 2020; revised accepted 23 November 2020

doi: $10.18520 /$ cs/v120/i4/637-643 